\title{
Every step you take
}

\author{
Walk more and sit less: even light exercise is linked to a lower risk of death
}

\author{
Jochen Klenk professor ${ }^{1}$, Ngaire Kerse professor ${ }^{2}$ \\ ${ }^{1}$ Institute of Epidemiology and Medical Biometry, Ulm University, Helmholtzstrasse 22, 89081 Ulm, Germany; ${ }^{2}$ Department of General Practice and \\ Primary Health Care, University of Auckland, Auckland, New Zealand
}

In the 1950s the London busman's study found that bus drivers developed a higher rate of coronary heart disease than their bus conductor counterparts. ${ }^{1}$ Since then, observational studies have repeatedly suggested that sedentary behaviour is bad and physical activity is good for health and longevity. Guidelines recommend at least 150 minutes of moderate intensity or 75 minutes of vigorous intensity aerobic physical activity each week. $^{23}$

Guideline evidence is mostly based on self report of the amount, intensity, and frequency of activity. Self report is, however, open to recall and reporting bias, potentially resulting in underestimation of low intensity activity and overestimation of overall activity. ${ }^{4}$ Self reports are also imprecise. Exactly how much activity (and at what intensity) is needed to protect health remains unclear. In addition, sedentary behaviour is emerging as a potentially independent risk factor for adverse health outcomes ${ }^{5}$ despite inconsistency in ascertainment of sedentary time. ${ }^{6}$

The introduction of body-worn sensors in the past decade has enabled more objective and precise data on the amount and intensity of physical activity and considerably advanced the specialty. But inconsistency and uncertainty remain, especially about the magnitude of any effects and the contribution to health of low levels of physical activity.

The systematic review and meta-analysis by Ekelund and colleagues (doi:10.1136/bmj.14570) combines high quality studies analysing the effect of sensor measured physical activity and sedentary behaviour on mortality. ${ }^{7}$ By harmonising the methods and considering more than 36000 people, 240000 person years of follow-up, and more than 2100 events, the authors were able to differentiate between different levels of intensity—including low intensity physical activity—with sufficient statistical power.

The results show non-linear dose-response relations between all activity measures, including sedentary time, and all cause mortality in adults. More than 9.5 hours of daily sedentary behaviour, excluding sleeping time, was associated with a statistically significant increased risk of death. In contrast, mortality fell steeply as total volume of physical activity increased up to a plateau at 300 accelerometer counts per minute of wear time. A similarly steep decrease in mortality occurred with increasing duration of light physical activity up to a plateau of about 300 minutes per day.

The new meta-analysis clarifies previous findings and confirms that even light activity, such as walking, is beneficial. The observed effect sizes for physical activity and mortality were substantially larger than those reported previously, potentially because of the improved precision of measurement and reduction of variance.

Questions remain, particularly over whether the effect of physical activity continues above a certain threshold. Previous studies using step detection to quantify activity have also reported a plateau after an initially steep decrease in mortality. ${ }^{89}$ Furthermore, it is unclear whether the effect of activity simply adds up or whether the distribution and complexity of activity across the day or week is relevant. We do not know if sedentary behaviour and physical activity are independent factors or if they represent two sides of the same coin. New statistical approaches such as compositional analyses are required to explore these interdependencies. ${ }^{10}$

The current analysis assumes that physical activity levels remain constant over time, which does not reflect reality. Changes in the duration and intensity of physical activity occur over the whole life span. Longitudinal data and novel methods examining activity trajectories are needed. This is also important to eliminate the risk of reverse causation whereby disease causes reduced activity, not the other way around.

\section{Every step counts}

Besides these details, the clinical message for general practitioners, public health professionals, policy makers, and the public seems straightforward: every step counts and even light activity is beneficial. Developing ways to limit sedentary time and increase activity at any level could considerably improve health and reduce mortality.

Effective interventions include prescriptions for activity from primary care practitioners, particularly with community follow-up. ${ }^{11}$ Health coaches also show promise. ${ }^{12}$ Activity 
prescribing is cheaper than many pharmaceutical interventions for cardiovascular disease and more effective at improving quality adjusted life years. ${ }^{13}$

Increasing activity at the population level is challenging, and sustained behaviour change is the holy grail of primary care and public health. ${ }^{14}$ Walking is one promising target for intervention. ${ }^{15}$ It is simple, affordable (free), achievable even for older adults, and rarely contraindicated.

In conclusion, Ekelund and colleagues' findings are important and easy to interpret: we should all move more and sit less and should encourage others to do the same.

Competing interests: We have read and understood BMJ policy on declaration of interests and have no relevant interests to declare.

Provenance and peer review: Commissioned; not peer reviewed.

Morris JN, Heady JA, Raffle PA, Roberts CG, Parks JW. Coronary heart-disease and physical activity of work. Lancet 1953;262:1053-7.

10.1016/S0140-6736(53)90665-5. 13110049

2 World Health Organization. Global recommendations on physical activity for health. WHO 2010.

32018 Physical Activity Guidelines Advisory Committee. Physical Activity Guidelines for Americans. 2nd ed. U.S. Department of Health and Human Services, 2018: 118.

4 Paul DR, McGrath R, Vella CA, Kramer M, Baer DJ, Moshfegh AJ. Understanding the Nature of Measurement Error When Estimating Energy Expenditure and Physical Activity via Physical Activity Recall. J Phys Act Health 2018;15:543-9. 10.1123/jpah.2017-0089. 29580135

5 Jefferis BJ, Parsons TJ, Sartini C, etal . Objectively measured physical activity, sedentary behaviour and all-cause mortality in older men: does volume of activity matter more than pattern of accumulation?Br J Sports Med 2019;53:1013-20 10.1136/bjsports-2017-098733. 29440040

6 Stamatakis E, Ekelund U, Ding D, Hamer M, Bauman AE, Lee I-M. Is the time right for quantitative public health guidelines on sitting? A narrative review of sedentary behaviour research paradigms and findings. Br J Sports Med 2019;53:377-82 10.1136/bjsports-2018-099131. 29891615

7 Ekelund U, Trap J, Steene-Johannessen J, etal . Dose-response associations between accelerometry measured physical activity and sedentary time and all cause mortality: systematic review and harmonised meta-analysis. BMJ 2019;366:14570.

8 Klenk J, Dallmeier D, Denkinger MD, Rapp K, Koenig W, Rothenbacher DActiFE Study Group. Objectively Measured Walking Duration and Sedentary Behaviour and Four-Year Mortality in Older People. PLoS One 2016;11:e0153779.

10.1371/journal.pone.0153779. 27082963

9 Lee I-M, Shiroma EJ, Kamada M, Bassett DR, Matthews CE, Buring JE. Association of Step Volume and Intensity With All-Cause Mortality in Older Women. JAMA Intern Med 2019; published online 29 May. 10.1001/jamainternmed.2019.0899. 31141585

10 Chastin SFM, Palarea-Albaladejo J, Dontje ML, Skelton DA. Combined Effects of Time Spent in Physical Activity, Sedentary Behaviors and Sleep on Obesity and Cardio-Metabolic Health Markers: A Novel Compositional Data Analysis Approach. PLoS One 2015;10:e0139984. 10.1371/journal.pone.0139984. 26461112

11 Elley CR, Kerse N, Arroll B, Robinson E. Effectiveness of counselling patients on physical activity in general practice: cluster randomised controlled trial. BMJ 2003;326:793. 10.1136/bmj.326.7393.793. 12689976

12 Rethorn ZD, Pettitt CD. What Is the Effect of Health Coaching Delivered by Physica Therapists? A Systematic Review of Randomized Controlled Trials. Phys Ther 2019;pzz098. 10.1093/pt//pzz098. 31309976

13 Garrett S, Elley CR, Rose SB, O'Dea D, Lawton BA, Dowell AC. Are physical activity interventions in primary care and the community cost-effective? A systematic review of the evidence. Br J Gen Pract 2011;61:e125-33. 10.3399/bjgp11X561249. 21375895

14 Rütten A, Frahsa A, Abel T, etal . Co-producing active lifestyles as whole-system-approach: theory, intervention and knowledge-to-action implications. Health Promot Int 2019;34:47-59. 10.1093/heapro/dax053. 28973298

15 Kelly P, Kahlmeier S, Götschi T, etal Systematic review and meta-analysis of reduction in all-cause mortality from walking and cycling and shape of dose response relationship. Int J Behav Nutr Phys Act 2014;11:132. 10.1186/s12966-014-0132-x. 25344355

Published by the BMJ Publishing Group Limited. For permission to use (where not already granted under a licence) please go to http://group.bmj.com/group/rights-licensing/ permissions 